\title{
A Systematic Literature Review of How News Games Explore a New Path in the Cross-Cultural Communication
}

\author{
Guangyao Jiang ${ }^{1, *}$, Meilin Zhi $^{2}$ \\ ${ }^{1}$ School of Communication \& Creative Arts, Faculty of Arts \& Education, Deakin University, Victoria, Australia \\ ${ }^{2}$ School of cultural\& communication, Zhejiang Wanli University, Zhejiang, China \\ *Corresponding author. Email: jianggu@deakin.edu.au
}

\begin{abstract}
The concept of news games has been proposed for some time. However, with recent developments in mass media technologies and facilities, there is increasing attention on the concept of news games as a novel way of presenting news. At the same time, research into news games has also undergone many changes and developments. This article analyzes the interactive relationships between news games and their users from the perspective of news communication and related literature. The main goal is to identify the application values of news games in the field of transcultural communication, particularly the element of high user interaction levels. A review of related literature indicates that the interactive relationships between news games and its users can be characterized by programming rhetoric, non-linear narratives, immersive experiences, soft communication through symbols to achieve emotional resonance, and so on. News games can provide a virtual space that tolerates various cultural elements, engage users from different cultural clusters to go beyond cultural barriers, as well as discuss and strengthen their understanding of news events.
\end{abstract}

Keywords: news game, cross-cultural communication, immersive reporting, interactive experience.

\section{INTRODUCTION}

According to the 2015 Nine Top Trends in Newsrooms, one new trend in media convergence is the gamification of news - the combination of video game technology with traditional news reporting [1]. News games present the news through digital technology, mainly through mobile media and can be fun, which accounts for their popularity on social media platforms [2]. By integrating multimedia and news programming, news scenarios create a virtual world [3]. In the process, news is disseminated by allowing audiences to participate by role-playing in the news events [4].

This interactive and immersive experience is the biggest difference between gamified and traditional news. News games allow viewers to enter a common virtual space or scene where the game leads them to seek and establish strong emotional resonance within that space. The entire experience is a cross-cultural process that weakens the influence of ideology by engaging users in the active construction of a "common meaning space" through empathy [5].

The news game is a novel method of news expression that enables audiences to understand and spread news information in the process of interaction, participation, and control, all of which upgrade the general experience of receiving information with a higher level of engagement. As a new way of sharing information, news games represent huge audience potential and untapped media market.

At the same time, news games have always been controversial. For example, the entertaining nature of news games can diminish the significance of news events, particularly serious issues such as disasters and politics. Moreover, shortcomings in the production and dissemination of news games include poor production efficiency, low cost-effectiveness, and limited dissemination channels. In addition, there is an insufficient degree of integration of news content and game-based design $[4,6]$. 
Meanwhile, research on news games remains at the cognitive level. While the basic concepts and characteristics of news games have been discussed, the significance of the wider uses of news games is still being debated. Moreover, there are no systematic analyses or solutions for the controversies and shortcomings of news games, nor are there practical suggestions related to the deep integration of news and game-based thinking.

Meanwhile, as a type of soft media that invites audiences to actively participate and understand news, news games in mobile communication has potentially strong cultural adaptability and communication power. In addition, in the field of cross-cultural communication, news games can change ways of discourse, help people break the cognitive barriers of cross-cultural communication, promote various cultural groups to reach social consensus, and provide new opportunities to enhance the power of international communication [7]. Thus, it is of critical significance to further explore how news games can provide new ideas for crosscultural communication in line with mass media development trends.

This paper summarizes current research on news games, its definitions, and key characteristics. At the same time, based on specific cases and the international communication environment, this paper aims to explore the relationships between news games, cross-cultural communication, and their development trends.

\section{INTERACTIVE RELATIONSHIPS BETWEEN NEWS GAMES AND AUDIENCES AND THE CROSS- CULTURAL COMMUNICATION OF NEWS GAMES}

\subsection{An Overview of the Characteristics and Significance of Contemporary News Games}

Researchers at home and abroad have not reached a consensus on the definition of news games. The three most widely spread viewpoints on the essence of news games are: 1) it is procedural rhetoric, 2) it is serious gaming, and 3) it is news.

For instance, Bogost et al., (2010) pointed out that a news game is essentially procedural rhetoric that simulates news stories by providing a game model that can interact with users [8]. While Chinese researchers also articulated similar views, they regard news games as serious and created according to journalism principles. For instance, Huang Mingfen believes that, in the digitalized world, news games apply "the principles of journalism to develop the media functions of games, and to provide players with virtual experiences based on real-world resources, usually real events and problems" [3], and that news games are "serious games closely related to news reports" [9].

Meanwhile, others define news games as essentially a new way of news reporting. According to Liu Yanlong, news games are "a news narrative mode characterized by strong interactivity" [10]. In addition, Jiang Yiting, producer of the Life in Emergency Department news game, points out that "news games are still about news in the final analysis".

Other researchers try to transcend this binary exchaNGE. For example, Jiang Xiaoli shows the different attributes of news and games based on different themes and narrative types in news games by defining two types of news games: "gamified news" and "news games" [11]. To further clarify the concept, Zhu Ai'min and $\mathrm{He}$ Lingling agree that "news game products created by professional game companies are serious games with stronger game attributes" while "news game works produced by news media are gamified news featuring more significant news attributes" [12].

In related studies, news games are widely placed within the scope of news coverage and studied as a type of news narrative technique. Moreover, the characteristics and communication effects of news games are explored and defined based on the features of news.

For instance, Kristin Breivik believes that news games can encourage players to experience the news on a more personal level and, on this basis, develop their own views on current events [13]. In a related analysis, Zhang Jianzhong points out that, audiences can use gaming as a medium to explore news events and participate in discussions related to the news, thus strengthening audience engagement with news events as well as enhancing their understanding of the significance of news events [14]. To sum up, as an innovative form of news delivery, news games have attracted wide attention in academia due to features such as active participation, immersive experience, multisequence narratives, and viral transmission [15].

Through multimedia integration and procedural narrative techniques, news games maximize the social context by providing realistic scenes. For instance, the first-person narrative and the role-playing plot create an immersive atmosphere where users can subjectively experience the news and, by strengthening the sense of substitution, strike a sympathetic chord in their hearts [16]. Moreover, through task completion modes, news games can create empathic experiences when the narrative switches from an onlooker perspective to a first-person perspective [17]. At the same time, with the help of virtual scenes, news games construct "common emotions", thus playing a powerful social mobilization role in inspiring and guiding public opinion. 
In short, news games provide audiences with immersive experiences by creating virtual reality scenes through multimedia technology and program rhetoric. In addition, with non-linear narration and interactive information dissemination, news games motivate audiences to participate, discuss news events, and strengthen their understanding of news events.

As concluded by Zhang Hui (2018), one key difference between gamified and traditional news reports is that news games can construct social situations and reflect social realities by disseminating specific information, thus providing virtual social experiences to users. Such an immersive communication environment and profound interactive experience are the core characteristics of news games [16] that regular news reports do not have.

There are still other controversies related to news games. For example, will news games turn serious news such as politics and disasters into entertaining news? Will the value scales produced by the audiences while understanding news events through games pose challenges in the guidance of public opinion? In addition, there are no currently unified standards concerning the production cost and timeliness of news games.

\subsection{How users becomes a cyberman in the common meaning space}

Bury Me, My Love won the Best Meaningful Play Award at the 14th International Mobile Gaming Awards (IMGA). This is a news game that featured text and adventure on the topic of Syrian refugees, focusing on the life of an ordinary Syrian couple in the midst of social unrest. The game includes two key characters: Nour, and her husband Majd.

A player takes on the role of Majd and communicates with Nour through text messages to help her as she takes refuge in Europe due to avoid the war. Each choice that the player makes can lead to nearly 50 different endings for Nour. The game allows players to make choices where they can feel the fear of refugees on the run and the anxiety of the loved ones they have left behind. Moreover, in the game, users can feel that every little choice they make could cost the life of the game's main character. In the process of completing this news game, the player gets to know the life of refugees, reflect on their wartime experiences, and care for the common destiny of mankind.

Yue Xiaoling's in-depth analysis of the news game discussed its production logic and pointed out a series of innovations used in the game. For instance, in terms of reporting, the game adopts a compound narrative strategy. In other words, a non-linear narrative technique from the first-person perspective is used to vividly present news events through sound and visual elements.

In addition, "choosing fate" through interactive means allows gamers an immersive experience in the process of receiving news and information. In the use of emotions common to human beings, the game deepens emotional resonance in players and, at the same time, achieves social education and enlightens the audience.

The main game scenario simulates the WhatsApp chat interface. To amplify the sense of entertainment and realism, players can send memes, pictures, links, voices, and more. As Yue pointed out, "news games weren't created to please people. Rather, we want to create an immersive news experience by connecting emotions through games and making each player a participant in the news" [15].

In terms of content, Yue said, the game brings the news to life with a selected soundtrack, friendly cartoons, signs, symbols and other figurative compensatory media to supplement text messages and elicit emotions and resonance among users [15]. For example, figurative elements such as the romance of the hero and heroine, voice, text message and video are all used. Yue points out how these figurative compensatory media can influence audience emotions and touch on the emotions common to all mankind by using various elements including words, ideas, senses and imagination, all of which are conducive to breaking down the cognitive barriers formed by the different cultural backgrounds and social identities of the users [15]. For example, there are stereotypes about Syrian refugees that can be transcended by playing the game.

In this news game, people are no longer news spectators but participants in the news events. The game leads the audience to think deeply about the events in the news, understand refugees, reflect on war, and care about the common destiny of mankind. In the process, the game taps into human emotions to build social empathy, which builds social consensus about the desire for peace.

In 2020, Shen Zhengrong and Wang Yuting posited similar views on the interactive effects caused by news games. They point out how this virtual situation turns news game users become "cybermen" in symbiosis with news games across time and space [7]. From a crosscultural communication perspective, when the game users share the same identity and fate, the interactive operation experience of the news games can smooth out the cultural and identity differences among the cybermen [7]. 


\subsection{News Games and Cross-Culture Communication}

As mentioned, news games allow users to share virtual situations and discourse networks in the games. Thus, the users can break down the discourse barriers of international communication both spatiotemporally and in the dimension of cultural differences, and then build a discourse community that transcends linguistic and cultural differences.

Moreover, as cybermen in news games, the users can transform themselves into a specific node or medium in the international communication of news games. This enables audiences to play active roles in news communication.

News games are vitalized in the context of international communication. As Shen \& Wang point out, news games can help strengthen international communication capabilities and create conditions that are favorable for the development and innovation of international communication. In news games, communication practitioners and news users from all over the world can reach a consensus in its scene interactions. In this scenario, a discourse community of global communication is built and China's soft cultural power and international communication capability can be enhanced [7].

In a similar vein, Jin Tiandong and Ren Xiao suggest that news games are designed to contribute to the improvement of the international discourse mode because a space of common meaning is created in the scene interaction, which can result in mutual understanding. They point out the necessity of creating such "a common meaning space" so as to reduce the communication barriers that prevent news from being effectively spread in the international community. With this space, news can reach wider and even more diverse audiences [18].

Shen Zhengrong and Wang Yuting note that news games have shown great vitality and innovation in the new media environment with technological empowerment and diversified communication subjects. Considering that, news games combine news and entertainment, attract users with strong interactivity and high participation, as well as use flexible communication strategies to transmit the news core [7].

As mentioned, news gamification is a flexible communication strategy that uses scene interaction and audience presence to break the cultural barriers and spatiotemporal limitations of international communication, which can, in the era of media convergence, provide new ideas and empowerment strategies for the breakthrough and development of international communication [7]. "To compete for the right to speak in the international public opinion environment with information globalization, international communication must seize the new opportunities brought by the emerging media technology and innovatively develop the form of news as inspired by news games" [7].

Li Jianjun et al. pointed out the penetrability and infectivity of flexible communication, which "often reflects the value orientation of communication and shapes positive images of a country through the content of cultural stories" [19].

In a word, with their strong empathy and concrete situational constructs, news games can build international discourse communities. To reprise, news games eliminate the spatiotemporal distance and cultural differences through the physical presence of user operation and interactions.

The communication mode that features scene interaction provides Chinese images and stories with more attraction and communicative elements that strengthen the validity and breadth of international communication. Moreover, the situational narrative, the first-person perspective, and the audio-visual combination provide news game users with an immersive experience so that they can gain firsthand experience of Chinese stories and culture.

In addition, as a flexible communication strategy, news games can show Chinese culture to the world in a gentle and friendly manner to build a discourse community that overcomes language and cultural barriers in the context of globalization, so as to firmly and effectively communicate Chinese culture around the world.

\section{CONCLUSION}

This article systematically reviews the interactive relationships between users and news games. A review of previous studies was aimed to determine the functions and significance of news games in transcultural communication.

From the news communication perspective, this article intends to present common methods and effects of the interaction between news games and users. In addition, through the analysis of classic cases, the development of the experience and research related to transcultural communication can be described.

In general, news games can help users immerse themselves unconsciously in common virtual scenes and social roles. Meanwhile, news games can provide unique interactions that can guide users so that they can actively overcome time and space limitations, cultural differences, social barriers, and establish emotional resonance with common consciousness. Not only does this accord with the experimental logic of transcultural 
communication, but it also opens new opportunities for international communication.

However, after a review of the related literature, this author found that current studies on news games and transcultural communication still focus on definitions, characteristics and significance of news games, and lack deep insights into specific aspects, particularly on the topic of international communication.

It is urgent that scholars should study typical cases of news games and systematically summarize news game design experience and strategies that can adapt to the era of multicultural mobile media. This author believes that designing and making good use of news games can provide important research contents that can further enhance China's international communication ability.

\section{REFERENCES}

[1] J.Z. Zhang, J. Posetti, A. Gezi, Nine top trends in newsrooms of 2015, Youth Journalist (22) (2015) 85-86. https://doi.org/10.15997/j.cnki.qnjz.2015.22.038

[2] Y.Q. Chang, Research on the spread of news games from the perspective of scene dissemination, Unpublished master's thesis, Southwestern University of Finance And Economics, 2019.

[3] M.F. Huang, News games in the digital context, Journal of Chongqing University of Posts and Telecommunications (Social Science Edition) 26(5) (2014) 94-100. https://doi.org/10.3969/j.issn.16738268.2014.05.019

[4] R.X. Jia, When the news "encounter" game: The concept, evolution and ethical thinking of the news game, North Media Research (3) (2018) 43-45. https://doi.org/10.19544/j.cnki.bmyj.2018.0075

[5] Q.G. Guo, Communication course, People's University of China Press, 2011.

[6] X. Chen, News game: Concept, characteristics and development dilemma, Youth Journalist (12) (2021) $109-110$. https://doi.org/10.15997/j.cnki.qnjz.2021.12.050

[7] Z.R. Shen, Y.T. Wang, Building a new path for international communication through immersive interaction in news games, Media Observer (11) (2020) 81-87. https://doi.org/10.19480/j.cnki.cmgc.2020.11.014

[8] I. Bogost, S. Ferrari, B. Schweizer, Newsgames: Journalism at play, The MIT Press, 2010.

[9] J.Z. Zhang, Towards a new media convergence: When news meets games, Modern Communication
(Journal of Communication University of China)

38(11) (2016) 111-116.

https://doi.org/10.3969/j.issn.1007-

8770.2016.11.023

[10] Y.L. Liu, Research on news games in the perspective of mass communication game theory, Unpublished master's thesis, Hebei University, 2017.

[11] X.L. Jiang, R.Q. Jia, The definition of newsgame yard a property, Journalism and Mass Communication Monthly (1) (2018) 42-47. https://doi.org/10.15897/j.cnki.cn511046/g2.2018.01.007

[12] A.M. Zhu, L.L. He, Exploring the applicability and limitations of news games, Radio \& TV Journal (2) (2021)

$168-170$

https://doi.org/10.19395/j.cnki.1674246x.2021.02.083

[13] K. Breivik, Games combatting the dictatorship of entertainment: A study of the newsgaming phenomenon (INFOMEV1345), University of Bergen, 2008.

[14] J.C. Zhang, J.F. Li, Innovation and practice of localized news games in restarting news narrative yard, Contemporary Communication (6) (2016) $45-47$, https://doi.org/CNKI:SUN:DACB.0.2016-06-018

[15] X.L. Yue, The existence value and problems of news game reflection--Bury $\mathrm{Me}$, My Love as an Example, Media (19), (2020) 62-64. https://doi.org/CNKI:SUN:CMEI.0.2020-19-022

[16] H. Zhang, The construction and deconstruction of social context by news games, Unpublished master's thesis, Northwest University, 2018.

[17] Y.N. Pan, News games: Concepts, motivations and characteristics, Shanghai Journalism Review (9), (2016) 22-28. https://doi.org/10.16057/j.cnki.31$1171 / \mathrm{g} 2.2016 .09 .004$

[18] T.D. Jin, X. Ren, Research on the "common meaning space" of international communication of "community of human destiny", Social Science (2), (2021) 32-46. https://doi.org/10.13644/j.cnki.cn311112.2021.02.004

[19] J.J. Li, H.Q. Liu, J. Liu, Strong communication and flexible communication: A new direction of foreign communication, Journal of Northeast Normal University (Philosophy and Social Sciences) (3) (2014) 190-195. https://doi.org/10.16164/j.cnki.221062/c.2014.03.059 